\title{
Modelling a neural network using an algebraic method
}

\author{
Prompong Sugunnasil $^{\mathrm{a}, *}$, Samerkae Somhom ${ }^{\mathrm{a}}$, Watcharee Jumpamule ${ }^{\mathrm{a}}$, Natee Tongsiri $^{\mathrm{b}}$ \\ ${ }^{a}$ Faculty of Science, Department of Computer Science, Chiang Mai University, Chiang Mai, Thailand 50200 \\ ${ }^{b}$ Faculty of Science, Department of Mathematics, Chiang Mai University, Chiang Mai, Thailand 50200
}

${ }^{*}$ Corresponding author, e-mail: p.sugunnasil@gmail.com

Received 8 Oct 2012

Accepted $3 \mathrm{Feb} 2014$

\begin{abstract}
In this paper, a framework based on algebraic structures to formalize various types of neural networks is presented. The working strategy is to break down neural networks into building blocks, relationships between each building block, and their operations. Building blocks are collections of primary components or neurons. In turn, neurons are collections of properties functioning as single entities, transforming an input into an output. We perceive a neuron as a function. Thus the flow of information in a neural network is a composition between functions. Moreover, we also define an abstract data structure called a layer which is a collection of entities which exist in the same time step. This layer concept allows the parallel computation of our model. There are two types of operation in our model; recalling operators and training operators. The recalling operators are operators that challenge the neural network with data. The training operators are operators that change parameters of neurons to fit with the data. This point of view means that all neural networks can be constructed or modelled using the same structures with different parameters.
\end{abstract}

KEYWORDS: formal models, specification, system design method

\section{INTRODUCTION}

Neural networks have been widely used in various fields such as biology, economics, chemistry, and physics. Within each of these fields of study, they were also employed for different tasks such as classification, regression and clustering. Over the years, various structures and several methods of neural networks have been proposed to improve their performances. However, the ways in which neural networks have been studied were considered to be more horizontal, that is, they were designed for the specific case, than vertical, which would have seen neural networks being cumulatively studied ${ }^{1}$.

It has also been raised that the implementation of neural networks seems to follow a trial and error principle. The nature of neural network development was considered as unpredictable and unrepeatable ${ }^{2}$, not to mention that methods to design neural networks using the prior knowledge or hypotheses have yet to be established. Moreover, neural network simulators which were required to fine-tune neural networks themselves were designed to apply only to a specific type of neural network, despite the fact that each generic neural network model shares some of the elements which can be used as building blocks for constructing new neural networks ${ }^{3}$.

To improve the situation mentioned above, we need a formal definition of neural networks capable of describing them in terms of both their ingredients and their relations. We introduce our algebraic framework based on the concept of building blocks which offer an inherent property of reusability. This framework will also enable practitioners to train different neural networks using the same simulator. Moreover, our proposed approach naturally offers insights into the constructive point of view of each neural network.

Many researchers have developed a unifying framework which was able to encapsulate both the architectures and the operations of the artificial network. The existing research can be categorized as non-computational or computational models.

The non-computational model is a type of model which focuses only on the architecture of the neural network. Specifically, they focus on connections between neurons in the network. Normally, graph theory is used as a guiding principle for this type of formalism. For example, in Ref. 1, the architecture of the network was depicted in terms of components and their associations. The components of the neural network were grouped into two types: the statics, and the dynamics. The statics are a group of components that remain unaltered during the operation on the network such as interconnection scheme, whereas the dynamics are a group of components which vary during the operation. Beside the graph-based approach, the set-based technique was also used to model neural networks. An interesting work in this category was the 
$\mathrm{Z}$ notation introduced in Ref. 2.

On the other hand, the computational model includes both the architecture, and the computation. The core idea of this approach is to decompose neural networks into components where each of them cannot be modelled in separation from each other. For example, the tripartite model introduced in Ref. 4 includes the environment in which the network operates, the input/output which describes both pre- and postprocessing, and the core which is the computational unit of the network. In Ref. 3, a formal framework to describe the neural network called a neural abstract machine was proposed in order to provide a platform to study the properties of neural networks. The neural abstract machine is based on the concept of the algebraic methods which separates the data and the operation on the data. The formal framework for the spiking neural network is proposed in Ref. 5. The spiking neural network is decomposed into a finer entity, such as synapses, activation functions, and neurons. Moreover, the environment can be regarded as a node. A set of node connected to each other to form the network. The execution of the model is simply done by receiving the input through ports, evaluating the input, and sending output through ports.

Although existing methods can capture various aspects of neural networks, there are still many desirable attributes missing. For example, in Ref. 6, Caelli, Guan, and Wen studied neural network in terms of collections of subnetwork module. The result indicated that modular neural network can reduce the computational complexity. The modularity of neural networks implies that there are groups of neurons functioning both under and above other groups of neurons - a nested structure. In this work, we study neural networks from a bottom-up fashion using algebraic methods as a modelling tool. We have noticed that all of neural networks are unique but they possess some common characteristics. They may have different types of activation function or different kind of learning mechanism but they are still based on the same structure - the neuron. The module in the neural network could be formed by the connection between neurons. The patterns of relationships between the neurons are what differentiate one model from the other. Another component of neural networks is the operation on the neural network. Operations on neurons are also included in our model in order to enable the computation of the model. As a result, we can construct any type of neural networks or even develop a new model of neural networks for a specific purpose.

The remainder of this paper is organized as fol-

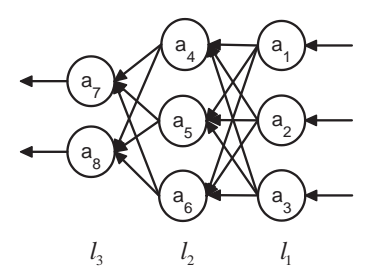

Fig. 1 A back-propagation network.

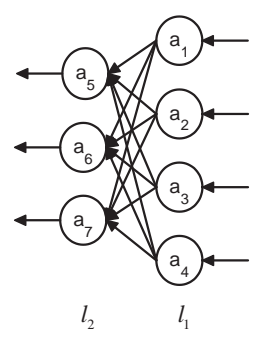

Fig. 2 A self-organizing map.

lows. In the next section, the method to model neural networks is elaborated which begins with the overall idea on how to model the neural network. Then, the proposed modelling method is provided in details. Each definition is elaborated with explicit examples. The last section concludes the study and discusses the possible further research directions.

\section{MODELLING NEURAL NETWORKS USING AN ALGEBRAIC METHOD}

In this section, we describe our proposed method. We first provide our concept of modelling the neural network using algebraic approach. Then, the definitions of the fundamental elements of our modelling approach are discussed including neuron, relationships between neurons, and operations on neurons. For each definition, we provide examples of the application of the definition of neural networks using the neural networks in Fig. 1, Fig. 2, and Fig. 3. Note that we do not define a specific activation function in our example. Throughout this work, $f: K \rightarrow K$ denotes the activation function.

The general strategy is to model neural networks

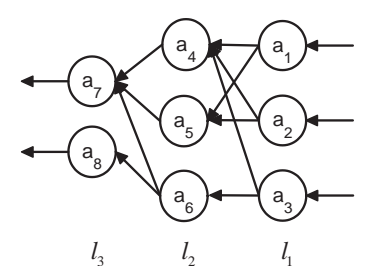

Fig. 3 A pruned neural network. 
using algebraic structures and methods. We combine the formalism of algebraic system with the bottom-up concept. Modelling using algebraic structure provides a bridge between the system requirement of the target system and its formal specification ${ }^{7}$. Also, modelling principles based on algebraic methods provide the means to systemically analyse the modelled system ${ }^{8}$.

In this work, the operation on a neuron is separated into two types. The first one is the structuring operator. This operator wires the neurons together to form a module of neurons. The second one is the operative operator. The operative operator is a computing operator which either uses the structure to perform calculation or adjusts the parameters of the structure. Finally, the component of the proposed method is combined into a single entity called the algebraic structure of neurons.

Throughout this paper, three neuron network models are used as an example for each definition. The back-propagation, and the self-organization map are displayed in Fig. 1, and Fig. 2, respectively. The third model displayed in Fig. 3 is the pruned version of the back-propagation.

The back-propagation is a well-known neural network with three staged training process; the feed forward of input signal, the calculation of error, and the update of the weights. In our example, there are eight components: five neurons, $a_{4}$ to $a_{8}$, and three input variables, $a_{1}$ to $a_{3}$.

For the neurons in the output layer, each output signal is compared against the corresponding target output signal in order to calculate the error information. The computation of error information is defined as

$$
\delta_{k}=\left(t_{k}-y_{k}\right) f^{\prime}\left(y_{i n_{k}}\right)
$$

where $\delta_{k}$ denotes the error information of the $k$ th output neuron, $t_{k}$ denotes the target output signal of the $k$ th output neuron, $y_{k}$ denotes the output signal of the $k$ th output neuron, and $y_{i n_{k}}$ denotes the activation value of the $k$ th output neuron.

The error information is then used to update the neuron of the output layer and propagates back to the hidden layer. The weight correction information is defined as

$$
\Delta w_{j k}=\mu \delta_{k} z_{j}
$$

where $w_{j k}$ denotes the weight connecting $j$ th hidden neuron and the $k$ th output neuron, $\mu$ denotes the learning rate, and $z_{j}$ denotes the output signal of $j$ th neuron. The weight correction information used to update the neuron is

$$
w_{j k}(\text { new })=w_{j k}(\text { old })+\Delta w_{j k}
$$

where $w_{j k}$ (new) and $w_{j k}$ (mathrmold) denote the updated weight connecting $j$ th hidden neuron and the $k$ th output neuron, the initial weight connecting the $j$ th hidden neuron and the $k$ th output neuron, respectively.

For the neurons in the hidden layer, the error information is obtained from the connected neurons in the higher layer. The incoming error information can be calculated by

$$
\delta_{i n_{j}}=\sum_{k=1}^{m} \delta_{k} w_{j k}
$$

where $\delta_{i n_{j}}$ denotes the incoming error information of the $j$ th neuron. The error information of the neuron is calculated by

$$
\delta_{j}=\delta_{i n_{j}} f^{\prime}\left(z_{i n_{j}}\right)
$$

where $\delta_{j}$ denotes the error information of the $j$ th hidden neuron. The weight correction information and the weight updating process are the same as the output layer.

Fig. 2 is an example of the self-organizing map an unsupervised neural network which brings out the underlying topological structure of the input file. The architecture of the self-organizing map consists of two layers; the cluster unit and the input unit. The cluster unit represents the topological structure of the input data which is typically one or 2-d. In this example, there are seven components including three neurons, $a_{5}$ to $a_{7}$, and four input variables, $a_{1}$ to $a_{4}$.

Unlike the back-propagation neural network, the self-organizing map is based on competitions. Each of the output signals is first compared with every cluster unit and only the cluster unit with the minimum distance is considered to be a winner. Moreover, not only the winning cluster unit is updated but also its neighbourhood cluster unit. The update function of the example is

$$
w_{i j}(\text { new })=w_{i j}(\text { old })+\mu\left(x_{i}-w_{i j}(\text { old })\right)
$$

where $w_{i j}$ (new) and $w_{i j}$ (old) denote the updated and initial weights connecting the $i$ th input neuron and the $j$ th cluster unit, respectively. $\mu$ denotes the learning rate, and $x_{i}$ denotes the $i$ th data value of the input pattern.

\section{PRELIMINARIES}

Definition 1 The universe of a neural network is a set that contains all of the possible values in a neural network. The universe of neural network is a ring $K$. 
Remark 1 We have not defined what the ring $K$ is. Basically, the universe of a neural network is a set that enables the operation between the components with some properties such as closure. Normally the universe of a neural network is a set of real numbers.

\section{NEURON AND LAYER}

In this study, the neuron is perceived as a collection of attributes, such as input channels, weights, activation functions, and learning rates. Moreover, the behaviour of a neuron is believed to be similar to a function by mapping between input and output. Essentially, the neuron is composed of an input channel, output channel, and activation function. Note that extra components such as radius of positive reinforcement, radius of negative reinforcement, and learning rate, can be included in the definition of the neuron later if it is necessary.

Definition 2 Given a universe of a neural network $K$, a set of outputs $K_{\text {out }} \subset K$, the arity $\tau \in \mathbb{N}$, and the activation function $f$ mapping from $\sum_{i=1}^{\tau} w_{i} x_{i}$ to $K_{\text {out }}$, a neuron is a function $a: K^{\tau} \rightarrow K_{\text {out }}$ which can be more elaborate by formalizing it as a threetuple $(X, W, \delta)$ where

(i) $X=\left\{x_{j} \mid x_{j} \in K \wedge j \in \mathbb{N} \wedge 0<j \leqslant \tau\right\}$ is a finite set called the input variables,

(ii) $W=\left\{w_{j} \mid w_{j} \in K \wedge j \in \mathbb{N} \wedge 0<j \leqslant \tau\right\}$ is a finite set called the weight, and

(iii) $f: K \rightarrow K_{\text {out }}$ is the activation function.

For simplicity, we denote the neuron by

$$
a\left(x_{1}, x_{2}, \ldots, x_{\tau}\right) .
$$

The set of neurons is denoted by $A$.

Example 1 The neuron in Fig. 1 and Fig. 2 can be modelled as $a=(X, W, f, \mu)$ where

(i) $X$ denotes a set of input variables. In this case, $X=\left\{x_{1}, x_{2}, x_{3}\right\}$ for $a_{4}, a_{5}, a_{6}, a_{7}$, and $a_{8}$ in Fig. 1 , and $X=\left\{x_{1}, x_{2}, x_{3}, x_{4}\right\}$ for $a_{5}, a_{6}$, and $a_{7}$ in Fig. 2,

(ii) $W$ denotes a set of weights where $W=$ $\left\{w_{1}, w_{2}, w_{3}\right\}$

(iii) $f$ denotes an activation function mappings from $\sum_{i=1}^{3} x_{i} w_{i}$ to $K_{\text {out }}$, and

(iv) $\mu \in K$ denotes a learning rate.

Definition 3 Given a universe of neural network, $K$, and a set of neurons, $A$, a layer is formally defined as a tuple $\left\langle t_{1}, \ldots, t_{n}\right\rangle$ where $t_{1}, \ldots, t_{n}$ are neurons, data, or input variables. The set of all layers is denoted by $W(A, K)$.
Example 2 In Fig. 1, there are three layers, $l_{1}$ to $l_{3}$. Each of the layers is formalized as follows. The purpose of this example is to demonstrate the appearance of the layer. Thus we only focus on the architecture of each layer. $l_{1}=\left\langle a_{1}, a_{2}, a_{3}\right\rangle, l_{2}=\left\langle a_{4}\right.$, $\left.a_{5}, a_{6}\right\rangle$, and $l_{3}=\left\langle a_{7}, a_{8}\right\rangle$.

The input data in the neural network are a layer whose members is all data from the universe of a neural network. The set of all data is denoted by a set $D=\left\{\left\langle d_{1}, \ldots, d_{p}\right\rangle \mid d_{1}, \ldots, d_{p} \in K \wedge p \in \mathbb{N}\right\}$.

\section{OPERATIONS ON NEURONS}

In this section, we introduce the operations on the neuron. There are two types of operation: recalling operation, and training operation. The training operation is a process of changing the parameters of the structure in relation to the training data. The training operation is modelled as a function mapping from a neuron to another neuron. The recalling operation is a process of challenging a neural network with a data, and yielding the output. In this work, the recalling operation is modelled as a function mapping from a neural network and data to data. The training operation is supervised or unsupervised. The supervised approach includes the target output signal to guide the training, while the unsupervised approach does not. Furthermore, the data are inherently given to the structure at the input channel which is represented by the input variables.

\section{Structuring operator}

The neurons can be assembled to function as a module in the neural network. In this paper, we call the module of neurons a structure. The structure in the neural network represents how information flows in the neural network. Additionally, structures can also coordinate with one another to construct a new structure. The types of behaviour of the structure are the same as the neurons' except that the end product of the structure's computation is a product of computation of the members in the structure. In this study, the essence of the structure is captured by using the composition of neurons where the outputs of neurons in the lower level functions as the inputs for the neurons in the higher level. In other word, the concept of the composition is used to model the flow of information in the neural network. The beneficial point in using the composition is that the layer can be designed in a separate manner.

As mentioned in Definition 3, there are two types of components existing at a time period, the input variable and the neuron. We perceive them as a term of the neuron. The term of neurons which 
combines the input variable and the neuron is denoted by $V(X, A)=X \cup A$.

Definition 4 Let $K$ be a universe of a neural network, $A$ be a set of neurons, and $W(A, K)$ be a set of layer. The composition of neuron and layer is given as

$$
S: V(X, A) \times W(A, K) \rightarrow V(X, A)
$$

by inductively setting the following steps. If $s=$ $x_{j}$ where $x_{j}$ denotes the $j$ th input variable, then $S\left(s,\left\langle t_{1}, t_{2}, \ldots, t_{n}\right\rangle\right)=t_{j}$. If $s=a\left(a^{1}, \ldots, a^{\tau}\right)$ and $a^{1}, \ldots, a^{\tau} \in A$, then $S\left(s,\left\langle t_{1}, t_{2}, \ldots, t_{n}\right\rangle\right)=$ $a\left(S\left(a^{1},\left\langle t_{1}, \ldots, t_{n}\right\rangle\right), \ldots, S\left(a^{\mathrm{r}},\left\langle t_{1}, \ldots, t_{n}\right\rangle\right)\right)$.

Definition 5 Let $K$ be a universe of neural network, $A$ be a set of neurons, and $W(A, K)$ be a set of layer. The composition of layers is given as

$$
\oplus: W(A, K) \times W(A, K) \rightarrow W(A, K)
$$

by setting $\left\langle t_{1}, \ldots, t_{n}\right\rangle \oplus\left\langle t_{1}^{\prime}, \ldots, t_{m}^{\prime}\right\rangle$ equal to $\left\langle S\left(t_{1},\left\langle t_{1}^{\prime}, \ldots, t_{m}^{\prime}\right\rangle\right), \ldots, S\left(t_{n},\left\langle t_{1}^{\prime}, \ldots, t_{m}^{\prime}\right\rangle\right)\right\rangle$.

Example 3 An illustration of composition between neurons in $l_{2}$ and the input variables in $l_{1}$ of the neural network in Fig. 1 is given as

$$
S\left(a_{4}\left(x_{1}, x_{2}, x_{3}\right),\left\langle a_{1}, a_{2}, a_{3}\right\rangle\right)=a_{4}\left(a_{1}, a_{2}, a_{3}\right) .
$$

An illustration of composition between neurons in $l_{3}$ and the neurons in $l_{2}$ is given by

$$
\begin{gathered}
S\left(a_{7}\left(x_{1}, x_{2}, x_{3}\right),\left\langle a_{4}\left(a_{1}, a_{2}, a_{3}\right), a_{5}\left(a_{1}, a_{2}, a_{3}\right),\right.\right. \\
\left.\left.a_{6}\left(a_{1}, a_{2}, a_{3}\right)\right\rangle\right) \\
a_{7}\left(a_{4}\left(a_{1}, a_{2}, a_{3}\right), a_{5}\left(a_{1}, a_{2}, a_{3}\right), a_{6}\left(a_{1}, a_{2}, a_{3}\right)\right) .
\end{gathered}
$$

The layer composition between layer $l_{1}$ and layer $l_{2}$ is given by

$$
\begin{gathered}
\left\langle a_{4}\left(x_{1}, x_{2}, x_{3}\right), a_{5}\left(x_{1}, x_{2}, x_{3}\right), a_{6}\left(x_{1}, x_{2}, x_{3}\right)\right\rangle \oplus \\
\left\langle a_{1}, a_{2}, a_{3}\right\rangle \\
=\left\langle a_{4}\left(a_{1}, a_{2}, a_{3}\right), a_{5}\left(a_{1}, a_{2}, a_{3}\right), a_{6}\left(a_{1}, a_{2}, a_{3}\right)\right\rangle .
\end{gathered}
$$

The total structure of the example in composition form is given by

$$
\begin{aligned}
& \left\langle a_{7}\left(x_{1}, x_{2}, x_{3}\right), a_{8}\left(x_{1}, x_{2}, x_{3}\right)\right\rangle \oplus \\
& \quad\left(\left\langle a_{4}\left(x_{1}, x_{2}, x_{3}\right), a_{5}\left(x_{1}, x_{2}, x_{3}\right), a_{6}\left(x_{1}, x_{2}, x_{3}\right)\right\rangle \oplus\right. \\
& \left.\quad\left\langle a_{1}, a_{2}, a_{3}\right\rangle\right) \\
& =\left\langle a_{7}\left(a_{4}\left(a_{1}, a_{2}, a_{3}\right), a_{5}\left(a_{1}, a_{2}, a_{3}\right), a_{6}\left(a_{1}, a_{2}, a_{3}\right)\right),\right. \\
& \left.a_{8}\left(a_{4}\left(a_{1}, a_{2}, a_{3}\right), a_{5}\left(a_{1}, a_{2}, a_{3}\right), a_{6}\left(a_{1}, a_{2}, a_{3}\right)\right)\right\rangle .
\end{aligned}
$$

Example 4 An illustration of composition between neurons in $l_{2}$ and the input variables in $l_{1}$ of the neural network in Fig. 2 is given by

$$
S\left(a_{5}\left(x_{1}, x_{2}, x_{3}, x_{4}\right),\left\langle a_{1}, a_{2}, a_{3}, a_{4}\right\rangle\right) .
$$

The total structure of the example in composition form is given by

$$
\begin{array}{r}
\left\langle a_{5}\left(x_{1}, x_{2}, x_{3}, x_{4}\right), a_{6}\left(x_{1}, x_{2}, x_{3}, x_{4}\right),\right. \\
\left.a_{7}\left(x_{1}, x_{2}, x_{3}, x_{4}\right)\right\rangle \oplus\left\langle a_{1}, a_{2}, a_{3}, a_{4}\right\rangle .
\end{array}
$$

Example 5 As an extension of the neural network in Fig. 1, we provide a more complicated neural network model by randomly pruning the network. The figure of the extended example is shown in Fig. 3. The total structure of example in composition form is given by

$$
\begin{gathered}
\left\langle a_{7}\left(x_{1}, x_{2}, x_{3}\right), a_{8}\left(x_{3}\right)\right\rangle \oplus \\
\left\langle a_{4}\left(x_{1}, x_{2}, x_{3}\right), a_{5}\left(x_{1}, x_{2}\right), a_{6}\left(x_{3}\right)\right\rangle \oplus\left\langle a_{1}, a_{2}, a_{3}\right\rangle \\
=\left\langle a_{7}\left(a_{4}\left(a_{1}, a_{2}, a_{3}\right), a_{5}\left(a_{1}, a_{2}\right), a_{6}\left(a_{3}\right)\right),\right. \\
\left.a_{8}\left(a_{6}\left(a_{3}\right)\right)\right\rangle .
\end{gathered}
$$

We list some of the fundamental properties of the composite neuron term. First, the composition of neural network is not commutative. The flow of information in the neural network is one direction. The order of the neurons contributes to the overall function of the network. Hence it is interchangeable. The composition of the neural network preserves the associativity. Within the same term of composition, the order of operations does not have an influence on either the performance or the result as long as the sequence is preserved.

The measurement of the structure's complexity is also examined in this paper. We identify the structure with trees. Typically, the tree is a connected graph without any cycles. The concept of complexity in terms of the tree is the height of a tree that represents the structure. Since the member of a certain structure is a nested structure of the layer, the complexity of the structure is determined through the maximum complexity of the member in the structure.

Definition 6 Let $a_{\tau}\left(t_{1}, \ldots, t_{\tau}\right)$ be a neuron. The depth of $a$ is determined by the maximum depth of its members. We formally define the depth of neuron's structure by

$$
\operatorname{depth}(a)=\max \left\{\operatorname{depth}\left(t_{1}\right), \ldots, \operatorname{depth}\left(t_{\tau}\right)\right\}+1
$$

where if $t=x \in X$ then $\operatorname{depth}(t)=0$, and if $t=a^{\prime}\left(t_{1}^{\prime}, \ldots, t_{\tau^{\prime}}^{\prime}\right)$, then $\operatorname{depth}(t)=$ $\max \left\{\operatorname{depth}\left(t_{1}^{\prime}\right), \ldots, \operatorname{depth}\left(t_{\tau^{\prime}}^{\prime}\right)\right\}+1$. 
Example 6 Consider the back-propagation neural network in Fig. 1, there are eight components: $a_{1}, a_{2}, a_{3}, a_{4}, a_{5}, a_{6}, a_{7}$, and $a_{8}$ where $a_{1}, a_{2}$, and $a_{3}$ are input variables, $a_{4}, a_{5}$, and $a_{6}$ are neurons in the hidden layer, and $a_{7}$, and $a_{8}$ are neurons in the output layer. Each of the neurons has its own input pattern. The depth of each component can be determined as $\operatorname{depth}\left(a_{1}\right)=\operatorname{depth}\left(a_{2}\right)=\operatorname{depth}\left(a_{3}\right)=0$, $\operatorname{depth}\left(a_{4}\right)=\operatorname{depth}\left(a_{5}\right)=\operatorname{depth}\left(a_{6}\right)=1$, and $\operatorname{depth}\left(a_{7}\right)=\operatorname{depth}\left(a_{8}\right)=2$.

\section{Operative operator}

The recalling operation is an operation to calculate the output signal of the input signal based on the parameter of the structure of neuron. In Definition 2, each neuron is already incorporates the activation function which enables the neuron to recall the data. Thus the definition of the recalling operation can focus solely on the behaviour. We give the formal definition of the recalling operation in terms of the composite form.

Definition 7 We say that a function $O_{\mathrm{r}}$ is a recalling operator, if $O_{\mathrm{r}}: A \times D \rightarrow K_{t}$ where $A$ denotes a set of neurons, $D$ denotes a set of data, and $K_{t} \subseteq K$ denotes a set of target output signal. Suppose that $a\left(t_{1}, t_{2}, \ldots, t_{\tau}\right)$ is a neuron with $\tau$ input variables. The recalling operator can be formally defined as

$$
\begin{aligned}
& O_{\mathrm{r}}\left(a\left(t_{1}, t_{2}, \ldots, t_{\tau}\right), d\right) \\
& = \begin{cases}f\left(\sum_{i=1}^{\tau} d_{i} w_{i}\right), & \operatorname{depth}(a)=0, \\
f\left(\sum_{i}^{\tau} O_{\mathrm{r}}\left(t_{i}, d\right) w_{i}\right), & \operatorname{depth}(a) \neq 0 .\end{cases}
\end{aligned}
$$

Definition $8 \mathrm{We}$ say that a function $O_{t_{s}}$ is a supervised training operator if $O_{t_{s}}: A \times D \times K_{t} \rightarrow A$ where $A$ denotes a set of neurons, $D$ denotes a set of data, and $K_{t} \subseteq K$ denotes a set of target output signal.

Example 7 In this example, the training operation of the back-propagation neural network in Fig. 1 and Fig. 3 is used as a demonstration. Basically, the weight updating process of our modelling method is the same as the traditional method except that it is rewritten in an algebraic representation. If the neuron is in the output layer, the weight of the neuron is updated by

$$
\begin{aligned}
& w_{j}(\text { new })=w_{j} \text { (old) } \\
& +\mu\left(\left(k-f\left(\sum_{i=1}^{\tau} w_{i} x_{i}\right)\right) f^{\prime}\left(\sum_{i=1}^{\tau} w_{i} x_{i}\right)\right)\left(x_{j}\right)
\end{aligned}
$$

where $w_{j}$ denotes the weight connecting the neuron and the $j$ th input, $\mu$ denotes the learning rate, $k$ denotes the target output signal, $f$ denotes the activation

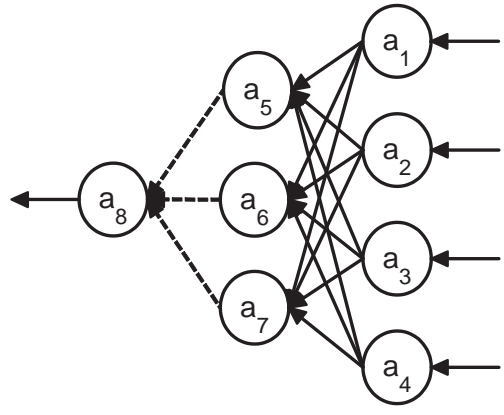

Fig. 4 A neural network model.

function, $f^{\prime}$ denotes the derivative activation function, and $x_{k}$ denotes the $k$ th input. If the neuron is in the hidden layer, the weight of the neuron is updated by

$$
w_{j}(\text { new })=w_{j}(\text { old })+\mu\left(\delta_{j} f^{\prime}\left(\sum_{i=1}^{\tau} w_{i} x_{i}\right)\right)\left(x_{j}\right)
$$

where $\delta_{j}$ denotes the sum of error information gathered from neurons in higher layers which uses information from the $j$ th neuron.

Definition 9 We say that a function $O_{t_{\mathrm{u}}}$ is an unsupervised training operator if $O_{t_{\mathrm{u}}}: A \times D \rightarrow A$ where $A$ denotes a set of neurons, and $D$ denotes a set of data.

Example 8 Like Example 7, the function of the neural network is preserved. The self-organizing map begins its weight updating process by determining the winner node. The winner node is the least different neuron compared to the input signal. Then, weights of both the winner node and its neighbour are updated. When the self-organizing map is updated, the process involves with not only the winner node but also the surrounding neurons. One way to solve the problem is to perceive the self-organizing map as a whole. Moreover, the process to determine the winner node is not the individual level process, but it is the whole level process which requires the information of every element. Hence the additional neuron has to be included to represent the entire neural network. In this case, the neuron $a_{8}$ is added to fill the requirement. The illustration of the new neural network is shown in Fig. 4. The weight training of the self-organizing map is given separately.

(i) If the neuron is the representation unit, the training process of this neuron is to call the recalling function on every neuron in the network and determines the winner node. Then, the representation unit performs the training operation on all of the neuron in the cluster unit. 
(ii) If the neuron is in the cluster layer, the weight of the neuron is updated using

$$
w_{j}(\text { new })=w_{j}(\text { old })+\mu h(j)\left(x_{j}-w_{j}\right)
$$

where $w_{j}$ is the $j$ th weight, $x_{j}$ denotes the $j$ th data, and $h(j)$ denotes the update information in relation to the distance from the winner node.

Definition 10 Let $W(A, K)$ denotes a set of layers, $A$ denotes a set of neurons, $D$ denotes a set of data, and $K_{t} \subseteq K$ denotes a set of target output signals. The extended supervised training operator is defined as

$$
\hat{O}_{t_{s}}: W(A, K) \times D \times K_{t} \rightarrow W(A, K)
$$

by setting

$$
\begin{array}{r}
\hat{O}_{t_{s}}\left(\left\langle t_{1}, \ldots, t_{n}\right\rangle,\left\langle d_{1}, \ldots, d_{n}\right\rangle, k\right)= \\
\left\langle O_{t_{s}}\left(t_{1},\left\langle d_{1}, \ldots, d_{n}\right\rangle, k\right), \ldots\right. \\
\left.O_{t_{s}}\left(t_{n},\left\langle d_{1}, \ldots, d_{n}\right\rangle, k\right)\right\rangle .
\end{array}
$$

Definition $11 W(A, K)$ denotes a set of layer, $A$ denotes a set of neurons, and $D$ denotes a set of data. The extended unsupervised training operator is given by

$$
\begin{gathered}
\hat{O}_{t_{\mathrm{u}}}: W(A, K) \times D \rightarrow W(A, K) \\
\text { by setting } \quad \hat{O}_{t_{\mathrm{u}}}\left(\left\langle t_{1}, \ldots, t_{n}\right\rangle,\left\langle d_{1}, \ldots, d_{n}\right\rangle\right)= \\
\left\langle O_{t_{\mathrm{u}}}\left(t_{1},\left\langle d_{1}, \ldots, d_{n}\right\rangle\right), \ldots, O_{t_{\mathrm{u}}}\left(t_{n},\left\langle d_{1}, \ldots, d_{n}\right\rangle\right)\right\rangle .
\end{gathered}
$$

Remark 2 In this study, we regard the order of the data given to the neural network as a training schema. The training schema is varied depending on the designer.

The set of operators is denoted by $O$.

\section{ALGEBRAIC STRUCTURE OF NEURONS}

Definition $12 A$ denotes a set of neurons, $D$ denotes a set of data, and $O$ denotes a set of operations. We define the neuron algebra using $\mathcal{A}=(A ; D, O)$ where

(i) $A$ is a set denoted the neurons,

(ii) $D$ is a set denoted the data, and

(iii) $O$ is a set denoted the operation on the neuron.

\section{CONCLUSION AND DISCUSSION}

Using the proposed framework, the artificial neural network can be designed in both directions: the topdown approach and the bottom-up approach. The network can be decomposed into several subnetwork modules due to their functions by focusing on the layer with the maximum depth, and moving down the network hierarchy. The neurons in the layer with the maximum depth represent the output layer of the network which is the function of the network. On the other hand, the designer can also focus the detail on the neurons in the layer with the maximum depth which represent the first group of the neurons which come in contact with the input. Consider

$$
\left\langle x_{1}^{1}, x_{2}^{1}, \ldots, x_{n_{1}}^{1}\right\rangle \oplus \ldots \oplus\left\langle x_{1}^{m}, x_{2}^{m}, \ldots, x_{n_{m}}^{m}\right\rangle .
$$

where the $x_{n}^{m}$ represents the $n$th neurons of the $m$ th layer. The top-down approach is to design the network from the first layer to the $m$ th layer, and the bottomup approach is to work from the $m$ th layer to the first layer. Moreover, since the proposed method uses the fundamental mathematical notation, the framework can be implemented in any formal language. The future extension of the proposed framework includes the incorporation of the temporal components which lead to a more expressive way to specify the artificial neural network.

Acknowledgements: The first author was supported by the Graduate School, Chiang Mai University. The fourth author was supported by the Department of Mathematics, Faculty of Science, Chiang Mai University.

\section{REFERENCES}

1. Fiesler E (1994) Neural network classification and formalization. Comput Stand Interfac 16, 231-9.

2. Senyard A, Kazmierczak E, Sterling L (2003) Software engineering methods for neural networks. In: Proceedings of the Tenth Asia-Pacific Software Engineering Conference Software Engineering Conference, IEEE Computer Society, Washington, DC, pp 468-77.

3. Borger E, Sona D (2001) A neural abstract machine. J Univers Comput Sci 7, 1006-23.

4. Dorffner G, Wiklicky H, Prem E (1994) Formal neural network specification and its implications on standardization. Comput Stand Interfac 16, 205-19.

5. Zaharakis ID, Kameas AD (2008) Modeling spiking neural networks. Theor Comput Sci 395, 57-76.

6. Caelli T, Guan L, Wen W (1999) Modularity in neural computing. Proc IEEE 87, 1497-518.

7. Goguen JA, Thatcher JW, Wagner EG, Wright JB (1977) Initial algebra semantics and continuous algebras. J ACM 24, 68-95.

8. Loegel G, Ravishankar CV (1994) An algebraic approach to modeling in software engineering. In: Proceedings of the 3rd International Conference on Methodology and Software Technology: Algebraic Methodology and Software Technology, Springer-Verlag, London, pp 385-92. 